\title{
CUSPIDAL HYPERGEOMETRIC FUNCTIONS
}

\author{
Eric M. Opdam
}

\section{Dedicated to Richard Askey on the occasion of his 65th birthday}

\begin{abstract}
We prove the Plancherel formula for hypergeometric functions associated with a root system when the root multiplicities are negative (but close to 0 ). As a result, we obtain a classification of the hypergeometric functions that are square integrable, and we find a closed formula for their square norm as a function of the root multiplicities.
\end{abstract}

\section{Introduction}

This section explains the main results of this paper (see the last two paragraphs of this section) and reviews some basic facts and notations of the theory of hypergeometric functions associated with root systems.

Let $\mathfrak{a}$ be a Euclidean space of dimension $n$ and $R \subset \mathfrak{a}^{*}$ (the dual of $\mathfrak{a}$ ) be an integral, irreducible, reduced root system which spans $\mathfrak{a}^{*}$. Its Weyl group is denoted by $W$. Let $\mathcal{K}$ denote the linear space of multiplicity functions, i.e., the space of $W$-invariant real functions on $R$. We fix a set of positive roots $R_{+}$. If $k \in \mathcal{K}$, we consider the following differential operator on $\mathfrak{a}$ :

$$
L(k)=\sum_{i=1}^{n} \partial\left(X_{i}\right)^{2}-\sum_{\alpha \in R_{+}} k_{\alpha}\left(1+e^{\alpha}\right)\left(1-e^{\alpha}\right)^{-1} \partial\left(X_{\alpha}\right) .
$$

Here we use the convention to write $\partial(p)$ (with $p$ an element of the symmetric algebra $\operatorname{Sym}(\mathfrak{a})$ of $\mathfrak{a})$ for the constant coefficient differential operator on $\mathfrak{a}$ that corresponds with $p$. Moreover, we have chosen an orthonormal basis $\left\{X_{i}\right\}$ of $\mathfrak{a}$, and $X_{\alpha} \in \mathfrak{a}$ is the element such that $\lambda\left(X_{\alpha}\right)=(\lambda, \alpha) \forall \lambda \in \mathfrak{a}^{*}$. This remarkable operator has been studied intensely over the years $[4,6-8,10-12,16,17]$. It arises "in nature" as the (radial part of the) Laplace-Beltrami operator of noncompact Riemannian symmetric spaces for special choices of the parameter $k$. It was realized that this operator plays a key role in the understanding of the Macdonald constant-term conjectures. Finally, this operator attracted some attention of mathematical physicists $[14,15]$ because of the relation of $L(k)$ with the quantum Calogero-Moser system. More precisely, define

$$
\rho(k)=\rho\left(R_{+}, k\right)=\frac{1}{2} \sum_{\alpha \in R_{+}} k_{\alpha} \alpha
$$

Received March 24, 1998, revised August 6, 1998.

1991 Mathematics Subject Classification: 33C80, 35P10, 46L45.

Key words and phrases: hypergeometric function, Plancherel formula, discrete spectrum. 
and

$$
\delta(k ; x)=\prod_{\alpha \in R_{+}}\left|2 \sinh \left(\frac{1}{2} \alpha(x)\right)\right|^{2 k_{\alpha}} .
$$

Then the operator $S(k)=\sqrt{\delta(k ; x)}(L(k)+(\rho(k), \rho(k))) \sqrt{\delta(k ; x)}^{-1}$ takes the form

$$
S(k)=\sum_{i=1}^{n} \partial\left(X_{i}\right)^{2}-\frac{1}{4} \sum_{\alpha \in R_{+}} \frac{(\alpha, \alpha) k_{\alpha}\left(k_{\alpha}-1\right)}{\sinh ^{2}\left(\frac{1}{2} \alpha(x)\right)} .
$$

This is the Schrödinger operator of the Calogero-Moser system. From this last formula, it is clear that $L(k)$ is a formally symmetric operator on the space $C_{c}^{\infty}(\mathfrak{a})^{W}$ if we equip this space with the Hermitian inner product

$$
(\phi, \psi)=\int_{\mathfrak{a}} \phi(x) \overline{\psi(x)} \delta(k ; x) d x
$$

where $d x$ denotes the Lebesgue measure on $\mathfrak{a}$. Moreover, we fix the length of the roots in such a way that a fundamental domain of the lattice $Q^{\vee}$ (here $Q^{\vee}$ is the coroot lattice in $\mathfrak{a}$, spanned over $\mathbf{Z}$ by the coroots $\alpha^{\vee}=\frac{2 X_{\alpha}}{(\alpha, \alpha)}(\alpha \in R)$ ) has volume equal to 1 (this turns out to be the most natural normalization as we shall see later). In this paper, we shall discuss the spectral problem for $L(k)$, in other words, we try to decompose the $L_{2}$ space of $W$-invariant functions associated with the measure in (1.5) as a direct integral of eigenspaces for $L(k)$. Formula (1.5) makes sense only as long as $\delta(k ; x)$ is a locally integrable function on $\mathfrak{a}$, and this imposes a condition on $k \in \mathcal{K}$. We shall restrict ourselves exclusively to this situation where $\delta(k ; x)$ indeed is locally integrable. Obviously, this condition is satisfied if $k_{\alpha}>0 \forall \alpha \in R$, and in this case, the spectral problem for $L(k)$ was solved completely in [17]. In this situation, the spectrum turns out to be completely continuous, and the spectral decomposition is very similar to the decomposition of the space $L^{2}(X)$ in irreducible (spherical) representations for $G$ if $X=G / K$ is a noncompact Riemannian symmetric space (cf. [6, 11]). The case that we are about to study in this paper is the one in which $k_{\alpha}<0 \forall \alpha \in R$, but satisfies condition (1.6) of Proposition 1.1 below so as to assure that $\delta(k ; x)$ is locally integrable. It is elementary to show that if $k_{\alpha}<0 \forall \alpha \in R$ and $\delta(k ; x)$ is locally integrable, then $\delta(k ; x)$ is integrable.

Proposition 1.1. Let $\beta \in R$ be the highest short root of $R$. If

$$
k_{\alpha}<0 \forall \alpha \in R \text { and } \rho(k)\left(\beta^{\vee}\right)+k_{\beta}+1>0 \text {, }
$$

then $\delta(k ; x)$ is integrable.

Proof. In [2], Corollary 2.2, the following sufficient condition was given: $\delta(k ; x)$ is integrable if

$$
k_{\alpha}<0 \forall \alpha \in R \text { and } \sum_{i=1}^{m} k_{i} h_{i}+1>0 .
$$

Here $h_{i}=\#\left(R_{i}\right) / n$ if the $R_{i}(i=1, \ldots, m)$ denote the $W$-orbits in $R$ (so $m=1$ or 2 ). If $m=1$, then this is equivalent to (1.6) because the height of the highest coroot equals $h_{1}-1$, as is well known. By the same formula, it is enough to check in the case $m=2$ that if $\sigma$ denotes half of the sum of the short positive roots, then one has $\sigma\left(\beta^{\vee}\right)=h_{1}-1$ if $R_{1}$ is the set of short roots. If $R_{1}$ is irreducible, this follows again 
from the above formula applied to $R_{1}$. If $R_{1}$ is reducible, then $R=B_{n}$, and here one verifies directly that $\sigma\left(\beta^{\vee}\right)=h_{1}-1=1$.

In the situation of Proposition 1.1, we shall give the spectral decomposition of the operator (1.1) almost explicitly, and, as we shall see, this involves lower dimensional spectral families. We can handle the situation by a combination of the techniques of [9] where we dealt with the so-called "Yang system" in the attractive case, and of [17]. In fact, it turns out that one has to adapt the arguments of these papers slightly at two critical points, and after that most of the arguments can be used. It is not our intention to repeat all the arguments of these papers. Instead, we shall just indicate the two critical steps mentioned above and, after that, freely use the statements of [9] and [17]. In any case, the results are quite nice. We obtain a complete classification of the square integrable eigenfunctions of $L(k)$, and we can, up to some rational constant, evaluate their square norms explicitly as a function of the multiplicities $k_{\alpha}$. Moreover, it seems likely that similar methods can be applied in even greater generality. For example, we have excluded the case $R=B C_{n}$ here because that really seems to call for more delicate arguments of a combinatorial nature. But that case is very interesting in its own right, and it should be investigated separately. Also, it seems likely that some form of the methods presented here should be applicable to the harmonic analysis of the so-called double affine Hecke algebra introduced by Cherednik. In this setting, one should obtain the Aomoto conjecture, recently proved by Macdonald [13], as the square norm computation of the simplest discrete eigenfunction.

In Section 2, we shall gather together some necessary notation and "basic" results about $L(k)$ and its eigenfunctions. In Section 3, we shall discuss the process of taking residues as it was formulated in [9] and explain how this applies to the situation in this paper. In Section 4, we prove the Paley-Wiener theorem, and finally, in Section 5 , we shall put things together and prove the main formula, the Plancherel formula.

\section{On the eigenfunctions of $L(k)$}

To describe the eigenfunctions of $L(k)$, we start by recalling one of the most important features of $L(k)$, namely that $L(k)$ is an element of a commutative algebra of $W$ invariant differential operators which is naturally isomorphic to $\operatorname{Sym}(\mathfrak{h})^{W}$ (here $\mathfrak{h}$ denotes the complexification of $\mathfrak{a}$ ). This fact can be understood in an elementary way using the following beautiful result about $L(k)$ due to Cherednik ([4]; also see [5, 7]). Define the so-called Cherednik operator

$$
D_{\xi}(k)=\partial_{\xi}+\sum_{\alpha \in R_{+}} k_{\alpha} \alpha(\xi) \frac{1}{1-e^{-\alpha}}\left(1-r_{\alpha}\right)-\rho(k)(\xi) .
$$

(Here $r_{\alpha}$ denotes the reflection in the root $\alpha$, and $\xi$ denotes an arbitrary element of $\mathfrak{h}$.) The following facts about the operators $D_{\xi}$ were discussed systematically in [17]. First of all, these operators map $C_{c}^{\infty}(\mathfrak{a})$ to itself (despite the apparent singularity in (2.1)). Next, the operators mutually commute and generate an operator algebra isomorphic to $\operatorname{Sym}(\mathfrak{h})$ where the isomorphism $\operatorname{Sym}(\mathfrak{h}) \ni p \rightarrow p(D)(k)$ is determined by the assignment $\mathfrak{h} \ni \xi \rightarrow D_{\xi}(k)$. Moreover, the $W$-invariant operators of the form $p(D)(k)$ are exactly those with $p \in \operatorname{Sym}(\mathfrak{h})^{W}$. Now we can restrict such $W$-invariant operators $p(D)(k)$ to $C_{c}^{\infty}(\mathfrak{a})^{W}$, and the resulting operator will be a differential operator mapping $C_{c}^{\infty}(\mathfrak{a})^{W}$ to itself. This differential operator will be denoted by $D_{p}(k)$. 
Finally $L(k)$ turns out to be an operator of this form:

$$
D_{\sum X_{i}^{2}}(k)=L(k)+(\rho(k), \rho(k)) .
$$

Let us now turn to the eigenfunction problem for the operators $D_{p}(k)$. For these facts, we refer to [17] and the references therein. The simplest eigenfunctions are the asymptotically free eigenfunctions $\Phi(\lambda, k ; x)$ on $\mathfrak{a}_{-}$. Indeed, if we assume that $\Phi(\lambda, k ; x)$ has an asymptotic expansion on $\mathfrak{a}_{-}$of the form ( $Q$ denotes the root lattice, i.e., the lattice spanned by the roots (over $\mathbf{Z}$ ))

$$
\Phi(\lambda, k ; x)=e^{(\lambda+\rho(k))(x)} \sum_{\kappa \in Q_{+}} \Delta_{\kappa}(\lambda, k) e^{\kappa(x)}
$$

with

$$
\Delta_{0}(\lambda, k)=1 \text {, }
$$

then all the coefficients are determined by recurrence by the single equation

$$
L(k) \Phi(\lambda, k ; x)=(\lambda+\rho(k), \lambda-\rho(k)) \Phi(\lambda, k ; x) .
$$

It is easy to see that $\Phi(\lambda, k ; x)$ automatically is a simultaneous eigenfunction for all the $D_{p}(k)$ :

$$
D_{p}(k) \Phi(\lambda, k ; x)=p(\lambda) \Phi(\lambda, k ; x) .
$$

Lemma 2.1. Let $\kappa \in Q$, but $\kappa \neq 0$. Then $\kappa^{\vee} \in C H\left(R^{\vee}\right)$, the convex hull of the coroots.

Proof. We may assume that $\kappa \in Q^{+}$and argue by induction on the height of $\kappa$. If the height of $\kappa$ equals 1 , the statement is obviously true. Next note that if the statement holds for two nonzero elements $\kappa_{1}$ and $\kappa_{2}$ in $Q^{+}$and $\left(\kappa_{1}, \kappa_{2}\right) \geq 0$, then the statement also holds for $\kappa_{1}+\kappa_{2}$. If $\kappa \in Q^{+}$and $\kappa \neq 0$, then there exists a simple root $\alpha$ such that $(\kappa, \alpha)>0$. If $(\kappa-\alpha, \alpha) \geq 0$, then we are done by the previous observation applied to the decomposition $\kappa=\alpha+(\kappa-\alpha)$. However, if $(\kappa-\alpha, \alpha)<0$, then $\kappa=r_{\alpha}(\kappa-\alpha)$ and we are done by the $W$-invariance of the problem.

We shall need the following facts about the asymptotically free eigenfunctions:

\section{Proposition 2.2.}

1. $\Delta_{\kappa}(\lambda, k)$ is a rational function, with poles at hyperplanes of the form $\lambda\left(\kappa^{\vee}\right)+1=$ $0\left(\kappa \in Q_{+} \backslash\{0\}\right)$ only.

2. Let $x_{0} \in \mathfrak{a}_{+}$, and $\varepsilon>0$. Then $\exists K_{x_{0}, \varepsilon} \in \mathbf{R}_{+}$such that $\forall \lambda$ with $\operatorname{Re}\left(\lambda\left(\alpha^{\vee}\right)\right)>\varepsilon-1$ $\forall \alpha \in R_{+}$, the following holds: $\forall \kappa \in Q_{+}$:

$$
\left|\Delta_{\kappa}(\lambda, k)\right| \leq K_{x_{0}, \varepsilon} e^{\kappa\left(x_{0}\right)}
$$

In particular, $\Phi(\lambda, k ; x)$ is analytic in $\lambda$ if $\operatorname{Re}\left(\lambda\left(\alpha^{\vee}\right)\right)>-1$ and $x \in \mathfrak{a}_{-}$.

Proof. See [17], Theorem 6.3. We have slightly relaxed the usual condition on $\lambda$ in 2 , and if one checks the proof of Gangolli in the classical context (cf. [11], Ch. IV, Lemma 5.6), it is clear that this is justified by Lemma 2.1 .

The next step is the introduction of the hypergeometric function for $R$, which is the unique (up to normalization) simultaneous eigenfunction of the $D_{p}(k)$ that extends holomorphically to a tubular neighbourhood of $\mathfrak{a}$ in $\mathfrak{h}$. We first need to define HarishChandra's $c$-function: 
Definition 2.3. For $\lambda \in \mathfrak{h}^{*}$ and $k \in \mathcal{K}$, we write

$$
\tilde{c}(\lambda, k)=\prod_{\alpha \in R_{+}} \frac{\Gamma\left(\lambda\left(\alpha^{\vee}\right)\right)}{\Gamma\left(\lambda\left(\alpha^{\vee}\right)+k_{\alpha}\right)}
$$

and

$$
c(\lambda, k)=\frac{\tilde{c}(\lambda, k)}{\tilde{c}(\rho(k), k)} .
$$

Theorem 2.4. ([8]; the different sign in the argument of the $c$-function is due to a change of sign in the definition of $\tilde{c}$.) Assume that $k \in \mathcal{K}$ satisfies (with $\beta \in R$ the highest short root)

$$
\rho(k)\left(\beta^{\vee}\right)+k_{\beta}+1>0
$$

(cf. (1.6)), that $\lambda$ is regular and that $\lambda\left(\kappa^{\vee}\right)+1 \neq 0(\forall \kappa \in Q \backslash\{0\})$. If $x \in \mathfrak{a}_{-}$, we define

$$
F(\lambda, k ; x)=\sum_{w \in W} c(-w \lambda, k) \Phi(w \lambda, k ; x)
$$

This function extends to a holomorphic function in $(\lambda, k, z)$ in the domain where $\lambda \in$ $\mathfrak{h}^{*}, k \in \mathcal{K}_{c}$ such that $\operatorname{Re}(k)$ satisfies (2.9), and $z=x+i y$ with $x, y \in \mathfrak{a}$ such that $|\alpha(y)|<2 \pi \forall \alpha \in R$. Moreover, $F(\lambda, k ; z)$ is $W$-invariant as a function of both $\lambda$ and $z$ and satisfies $F(\lambda, k ; 0)=1$.

Proof. This result is well known, cf. [8] or [17, Theorem 6.3]. Notice that one avoids the zeros of the $\tilde{c}(\rho(k), k)$ because of $(2.9)$.

Apart from the important asymptotic behavior with respect to $z$ of $F(\lambda, k ; z)$ given in Theorem 2.4, we want to have good control over the growth behavior in $\lambda \in \mathfrak{h}^{*}$ of $F(\lambda, k ; x)$ when $x$ is confined to a compact subset of $\mathfrak{a}$. This is of crucial importance for the Paley-Wiener theorem. In [17], we proved a uniform estimate in both $x$ and in $\lambda$, but in this strong form the result will no longer be true if $k_{\alpha}<0$. Fortunately, we can use the calculus of shift operators to prove a weaker statement that is just sufficient to prove the Paley-Wiener theorem.

Theorem 2.5. Assume that $k \in \mathcal{K}$ is such that $\tilde{c}(\rho(k), k) \neq 0$. Let $D \subset \mathfrak{a}$ be compact and let $p \in \operatorname{Sym}(\mathfrak{h})$. There exist constants $C \in \mathbf{R}_{+}$and $M \in \mathbf{N}$ such that $\forall \lambda \in \mathfrak{h}^{*}$, $\forall x \in D$ :

$$
|\partial(p) F(\lambda, k ; x)| \leq C(1+|\lambda|)^{M} e^{\max _{w} \operatorname{Re}(w \lambda(x))} .
$$

Proof. By a slight variation of [17], Corollary 6.2, we see that this result is true when $k_{\alpha} \geq 0 \forall \alpha \in R$. We now argue, inductively, using the following formula from the calculus of hypergeometric shift operators (the formula is similar in nature to formula (5.1) of [17], but we omit details):

$$
b(k) F(\lambda, k ; x)=\varepsilon_{+}\left\{\prod_{\alpha \in R_{+}} D_{\alpha^{\vee}}(k)(\Delta(x) F(\lambda, k+1 ; x))\right\}
$$

where $\varepsilon_{+}$denotes symmetrization over $W$ with respect to $x, k+1$ is the multiplicity function such that $(k+1)_{\alpha}=k_{\alpha}+1, \Delta$ denotes the Weyl denominator

$$
\Delta(x)=\prod_{\alpha \in R}\left(e^{\frac{\alpha(x)}{2}}-e^{\frac{-\alpha(x)}{2}}\right),
$$


and $b(k)=\frac{\tilde{c}(\rho(k), k)}{\tilde{c}(\rho(k+1), k+1)}$, which is a polynomial in $k$, nonvanishing under the assumption that $\tilde{c}(\rho(k), k) \neq 0$. We may assume that $D$ is $W$-invariant and convex. Now observe that

$$
\begin{aligned}
D_{\xi}(k) f(x)=\partial(\xi) f(x)-\rho(k) & (\xi) \\
& f(x) \\
& -\sum_{\alpha \in R_{+}} k_{\alpha} \frac{\alpha(x) \alpha(\xi)}{1-e^{-\alpha(x)}} \int_{t=0}^{1} \partial\left(\alpha^{\vee}\right) f\left(x-t \alpha(x) \alpha^{\vee}\right) d t .
\end{aligned}
$$

From this expression, we easily see that (2.10) holds for $F(\lambda, k, x)$, assuming that (2.10) holds for $F(\lambda, k+1 ; x)$.

\section{Wave packets and the tempered spectrum}

In the rest of this paper, we assume that the condition (1.6) holds, unless stated otherwise. Recall the definition of Paley-Wiener functions.

Definition 3.1. Given $x \in \mathfrak{a}$, let $C_{x}$ denote the convex hull of $W x$. Define the support function $H_{x}$ on $\mathfrak{a}^{*}$ as follows: $H_{x}(\lambda)=\sup _{y \in C_{x}} \lambda(y)$. An entire function $\phi$ on $\mathfrak{h}^{*}$ is said to have Paley-Wiener type $x$ if $\forall N \in \mathbf{Z}_{+} \exists C \in \mathbf{R}_{+}$such that

$$
|\phi(\lambda)| \leq C(1+|\lambda|)^{-N} e^{H_{x}(-\operatorname{Re} \lambda)} .
$$

The space of functions of Paley-Wiener type $x$ is denoted by $P W(x)$. We also use the notation $P W=\bigcup_{x \in \mathfrak{a}} P W(x)$.

We are going to study the following wave packet operator. If $\phi \in P W$, we take a point $p \in \mathfrak{a}_{+}^{*}$ such that

$$
p\left(\alpha^{\vee}\right)+k_{\alpha}>0 \quad \forall \alpha \in R
$$

and we define for $x \in \mathfrak{a}_{-}$:

$$
\mathcal{J}(\phi)(x)=(2 \pi i)^{-n}|W|^{-1} \int_{p+i \mathfrak{a}^{*}} \phi(\lambda) \Phi(\lambda, k ; x) \frac{d \lambda}{c(\lambda, k)} .
$$

We observe right away that this definition is independent of the choice of the point $p$ as long as $p$ satisfies the condition (3.1). First of all, the integrand is holomorphic in the domain $\operatorname{Re} \lambda\left(\alpha^{\vee}\right)+k_{\alpha}>0 \forall \alpha \in R$. Because of Proposition 2.2 (2), we have a uniform bound on $\Phi(\lambda, k ; x)$, so it is allowed to apply Cauchy's theorem by the Paley-Wiener estimates on $\phi$ and the following simple fact for the reciprocal of the $c$-function: if $k_{\alpha}<0 \forall \alpha \in R$, then $c(\lambda, k)^{-1}$ is bounded on regions of the form $\operatorname{Re} \lambda\left(\alpha^{\vee}\right)+k_{\alpha} \geq \epsilon>0$.

Next, let us consider what happens when we move $p$ towards the origin and thus cross the poles of $c(\lambda, k)^{-1}$. This process was studied in a somewhat simpler context in [9]. Fortunately, it is easy to reduce the situation to that of [9], so that we can use the results of that paper without difficulty. Let us first recall some important notions of [9] that are indispensable for the formulations of our results.

For $L \subset \mathfrak{a}^{*}$, an affine subspace, we put $R_{L}=\left\{\alpha \in R \mid L\left(\alpha^{\vee}\right)=\right.$ constant $\}$. If $\mathfrak{a}_{L}^{*}=\operatorname{span}\left(R_{L}\right)$, then it is clear that $R_{L}=R \cap \mathfrak{a}_{L}^{*}$ is a parabolic root subsystem of $R$. An affine subspace $L \subset \mathfrak{a}^{*}$ is defined to be residual (or, more precisely, $\left(\mathfrak{a}^{*}, R, k\right)$ residual) by induction on the codimension of $L$. The space $\mathfrak{a}^{*}$ itself is by definition a residual subspace. The affine subspace $L \subset \mathfrak{a}^{*}$ with positive codimension is called 
residual if there is a residual subspace $M \subset \mathfrak{a}^{*}$ with $M \supset L$ and $\operatorname{dim}(M)=\operatorname{dim}(L)+1$ such that

$$
\#\left\{\alpha \in R_{L} \backslash R_{M} \mid L\left(\alpha^{\vee}\right)=k_{\alpha}\right\} \geq \#\left\{\alpha \in R_{L} \backslash R_{M} \mid L\left(\alpha^{\vee}\right)=0\right\}+1
$$

A residual point also is called a distinguished (or, more precisely, $\left(\mathfrak{a}^{*}, R, k\right)$-distinguished) point. If $L \subset \mathfrak{a}^{*}$ is an affine subspace with $\operatorname{codim}(L)=\operatorname{rank}\left(R_{L}\right)$, then $L=c_{L}+V^{L}$ with $c_{L}$ the center of $L$ determined by $\left\{c_{L}\right\}=L \cap V_{L}$ and $V^{L}$ the orthogonal complement of $V_{L}$ in $V$. When $L$ is a residual subspace, we define the tempered form $L^{t e m p}=c_{L}+i V^{L} \subset \mathfrak{h}^{*}$. There is a complete classification of the residual subspaces for each root system; cf. [9], Section 4.

Lemma 3.2. For every residual subspace $L$, the center $c_{L}$ lies in the convex hull of the $W$-orbit of $\rho(k)$. In particular, $c_{L}$ satisfies $\left|c_{L}\left(\alpha^{\vee}\right)\right|<1 \forall \alpha \in R$.

Proof. We may assume that $c_{L}$ is dominant. Clearly, it is sufficient to prove the lemma for the situation $L=c_{L}$, i.e., the case when $L$ is a distinguished point. Let $\beta_{1}, \ldots, \beta_{N}$ be the positive roots such that $L\left(\beta_{i}^{\vee}\right)=k_{\beta_{i}}$. Using the classification of distinguished points of [9], we see that for every simple root $\alpha$, there exists an index $i$ such that $\beta_{i} \geq \alpha$ and $\left|\beta_{i}\right|=|\alpha|$ (if $R$ is simply laced, this is obvious; unfortunately, we do not see a uniform proof in the other cases). Hence, $L\left(\alpha^{\vee}\right) \leq-k_{\alpha}=-\rho(k)\left(\alpha^{\vee}\right)$ for every simple $\alpha \in R$, which is even a stronger statement than what we wanted to prove. The last statement of the lemma follows from Proposition 1.1.

The $c$-function has a nice decomposition in terms of the $c$-function of the Yang system which was introduced in [9]. Indeed, define

$$
c_{Y}(\lambda, k)=\prod_{\alpha \in R_{+}} \frac{\alpha^{\vee}(\lambda)+k_{\alpha}}{\alpha^{\vee}(\lambda)}
$$

and define $c^{Y}(\lambda, k)$ by the decomposition

$$
c(\lambda, k)=c_{Y}(\lambda, k) c^{Y}(\lambda, k) .
$$

We have:

Lemma 3.3. Let $U$ be a $W$-invariant open convex set that contains the convex hull of the $W$-orbit of $\rho(k)$ and which is itself contained in $\left\{\lambda \in \mathfrak{a}^{*}|\forall \alpha \in R:| \lambda\left(\alpha^{\vee}\right) \mid<\right.$ $\left.1+k_{\alpha}\right\}$. Both $c^{Y}(\lambda, k)$ and $c^{Y}(\lambda, k)^{-1}$ are holomorphic in the tube $U+i \mathfrak{a}^{*}$, and $c^{Y}(\lambda, k)^{-1}$ is bounded on this domain. Moreover, the product $c^{Y}(\lambda, k) c^{Y}(-\lambda, k)$ is $W$ invariant, and positive on every tempered subspace $L^{\text {temp }}$.

Proof. We can choose $U$ as indicated because of Proposition 1.1, and because of Lemma 3.2, we know that $L^{\text {temp }} \subset U+i \mathfrak{a}^{*}$ for every residual subspace $L$. The rest of the statements follow directly from the explicit formula

$$
c^{Y}(\lambda, k)=\tilde{c}(\rho(k), k)^{-1} \prod_{\alpha \in R_{+}} \frac{\Gamma\left(\lambda\left(\alpha^{\vee}\right)+1\right)}{\Gamma\left(\lambda\left(\alpha^{\vee}\right)+k_{\alpha}+1\right)} .
$$

The positivity is proved as in [9], Theorem 3.13. This is based on the fact that $-c_{L} \in W\left(R_{L}\right) c_{L}$, cf. [9], Theorem 3.10 .

With these preliminaries in mind, it is now clear that we can apply the residue calculus as formulated in [9], Section 3 , to the wave packet (3.2). Indeed, we can take $p$ in $U$ (see Lemma 3.3), still satisfying (3.1). By Lemma 3.2 and the fact that $U$ is 
convex and $W$-invariant, it is clear that the contour shifts needed in the calculation of residues all take place inside the tube $U+i \mathfrak{a}^{*}$. Therefore, all the poles of the integrand that one has to reckon with are those lying in the tube $U+i \mathfrak{a}^{*}$, and, by Proposition 2.2 and Lemma 3.3, these come only from $c_{Y}(\lambda, k)^{-1}$, the reciprocal of the $c$-function of the Yang system. To describe the result, we introduce the following function supported on $L^{t e m p}$. If $\lambda \in L^{t e m p}$, write

$$
f_{L}(\lambda, k)=\tilde{c}(\rho(k), k)^{2} \frac{\prod^{\prime}\left|\Gamma\left(\lambda\left(\alpha^{\vee}\right)+k_{\alpha}\right)\right|}{\Pi^{\prime}\left|\Gamma\left(\lambda\left(a^{\vee}\right)\right)\right|}
$$

where the $\Pi^{\prime}$ denotes the product over all roots such that the argument of the corresponding gamma factor is not identically equal to 0 on $L^{t e m p}$.

With these definitions, we have:

Theorem 3.4. Let $\omega_{L}$ denote the measure on $L^{\text {temp }}$ which is invariant under translations by elements of $i V^{L}$, normalized such that if $F$ is a fundamental domain of $V^{L} \cap 2 \pi P$ ( $P$ is the weight lattice of $R$ ), then the volume of $c_{L}+i F$ is 1 . If $\phi \in P W$ and is $W$-invariant, and $x \in \mathfrak{a}_{-}$, then $\mathcal{J}(\phi)(x)$ also can be written in the form

$$
\mathcal{J}(\phi)(x)=\int \phi(\lambda) F(\lambda, k ; x) d \nu(\lambda, k)
$$

where $\nu=\sum_{L} \nu_{L}$ (the sum taken over all the residual subspaces), and $\nu_{L}$ is a measure supported on $L^{\text {temp }}$ of the form

$$
\nu_{L}(\lambda, k)=\gamma_{L}(k) f_{L}(\lambda, k) \omega_{L}(\lambda) .
$$

Here $\gamma_{L}(k)$ is a nonnegative rational constant, depending on the configuration of hyperplanes of poles of $c_{Y}^{-1}$. It is $W$-invariant $\left(\gamma_{L}(k)=\gamma_{w(L)}(k)\right)$ and invariant for scaling $\left(\gamma_{L}(k)=\gamma_{L}(t k)\right.$ if $\left.t \in(0,1]\right)$. This constant is hard to compute in general, but the following cases are easy: $\gamma_{\mathfrak{a}^{*}}=|W|^{-2}$, and if $c$ is a regular distinguished point defined by $\beta_{i}^{\vee}(c)+k_{\beta_{i}}=0, i=1, \ldots, n,\left(\beta_{i} \in R\right)$ then there are two possibilities: if $c$ is a strictly positive combination of the roots $\beta_{i}$, then $\gamma_{c}=|W|^{-2}$ ind $^{-1}$ where ind is the index of the lattice spanned by the coroots $\beta_{i}^{\vee}$ in $Q^{\vee}$. Otherwise. $\gamma_{c}=0$. The function $f_{L}$ is smooth, nonnegative, and bounded on $L^{\text {temp }}$.

Proof. Everything follows by applying the steps in the proof of [9], Theorem 3.18 to

$$
\mathcal{J}(\phi)(x)=(2 \pi i)^{-n}|W|^{-1} \int_{p+i \mathfrak{a}^{*}} \frac{\phi(\lambda)}{c^{Y}(\lambda, k) c^{Y}(-\lambda, k)} c^{Y}(-\lambda, k) \Phi(\lambda, k ; x) \frac{d \lambda}{c_{Y}(\lambda, k)}
$$

and the fact that (Theorem 2.4)

$$
F(\lambda, k ; x)=\sum_{w \in W} c_{Y}(-w \lambda, k) c^{Y}(-w \lambda, k) \Phi(w \lambda, k ; x) .
$$

We omit the details concerning the normalizations of measures. This is simply a matter of making a careful comparison with the residue formulas in [9]. The statements about $f_{L}$ follow from Lemma 3.3 and formula (3.8), in combination with [9], formula (3.2) (and the remarks following formula (3.2)).

At this point we can deduce very powerful conclusions about the leading terms of hypergeometric functions, by application of [9], Corollary 3.7, and Corollary 3.8 to formula (3.11). We obtain: 
Corollary 3.5. If $\lambda \in \operatorname{supp}(\nu)$, then $F(\lambda, k)$ has a convergent asymptotic expansion on $\mathfrak{a}_{-}$of the form

$$
F(\lambda, k ; x)=\sum_{\mu \in W \lambda} \sum_{\kappa \in Q_{+}} p_{\mu, \kappa}(\lambda, k ; x) e^{\{\rho(k)+\mu+\kappa\}(x)}
$$

where the first sum is taken over $\mu \in W \lambda$ such that $\operatorname{Re}(\mu) \geq 0$ (in the usual dominance ordering). Here the $p_{\mu, \kappa}$ are polynomials in $x$. If $\{\lambda\}=L$ is a distinguished point and $\nu_{L}(L) \neq 0$, then this can be sharpened: the $\mu$ that are used in (3.12) have strictly positive real parts now.

Definition 3.6. The support of $\nu(\lambda, k)$ is called the tempered spectrum. If $\lambda=L$ is a distinguished point in the tempered spectrum, then we call $\lambda$ a cuspidal point.

We can apply the techniques of Casselman and Miličić [3] in order to obtain estimates for $F(\lambda, k)$ on $\mathfrak{a}$ if $\lambda$ is in the tempered spectrum.

Corollary 3.7. Let $\lambda$ be in the tempered spectrum, and choose $\tau$ in the closure of $\mathbf{R}_{+} R_{+}$such that $\tau \leq \operatorname{Re}(\mu)$ for all $\mu$ that occur in (3.12). Then $\forall x \in \overline{a_{-}}$, we have

$$
|F(\lambda, k ; x)| \leq M(1+|x|)^{m} e^{\{\tau+\rho(k)\}(x)}
$$

for suitable constants $M$ and $m$. In particular, if $\lambda$ is a cuspidal point, then $F(\lambda, k) \in$ $L_{p}(\mathfrak{a}, \delta(k, x) d x)$ for all $1 \leq p<2+\epsilon$ for some $\epsilon>0$.

\section{The Paley-Wiener theorems}

We are now in the position to prove the Paley-Wiener theorem. Given $f \in C_{c}^{\infty}(\mathfrak{a})^{W}$, we define its Fourier transform $\mathcal{F}(f)$ by

$$
\mathcal{F}(f)(\lambda)=\int_{\mathfrak{a}} f(x) F(-\lambda, k ; x) \delta(k ; x) d x .
$$

(Recall the normalization of the roots in formula (1.5).)

Theorem 4.1. If the support of $f \in C_{c}^{\infty}(\mathfrak{a})^{W}$ is contained in $C_{x}$ (the convex hull of $W x$ ) for some $x \in \mathfrak{a}$, then $\mathcal{F}(f) \in P W(x)$ (cf. Definition 3.1), and $\mathcal{F}(f)$ is $W$ invariant.

Proof. We use the fact that if $p$ is a real $W$-invariant polynomial on $\mathfrak{a}^{*}$, and $p^{\vee}$ denotes the polynomial $p^{\vee}(\lambda)=p(-\lambda)$, then

$$
\left(D_{p} f, g\right)=\left(f, D_{p^{\vee}} g\right)
$$

where $(\cdot, \cdot)$ is the inner product $(1.5)$, and $f$ and $g$ are in $C^{\infty}(\mathfrak{a})^{W}$, at least one of them having compact support (cf. Lemma 7.8 of [17]). Hence, by Theorem 2.5, for all real $W$-invariant polynomials $p$ on $\mathfrak{a}^{*}$, there exist constants $C$ and $M$ such that

$$
|p(\lambda)||\mathcal{F}(f)(\lambda)| \leq C(1+|\lambda|)^{M} e^{H_{x}(-\operatorname{Re} \lambda)}
$$

with $M$ independent of $p$. This proves the result.

We now proceed with the converse statement for the wave packet operator $\mathcal{J}$ discussed in the previous section.

Theorem 4.2. If $\phi \in P W(x)$ for some $x \in \mathfrak{a}$, and $\phi$ is $W$-invariant, then $\mathcal{J}(\phi) \in$ $C_{c}^{\infty}\left(C_{x}\right)^{W}$. 
Proof. First write $\mathcal{J}(\phi)$ in the form (3.8). Using the boundedness of the functions $f_{L}$ on $L^{\text {temp }}$ and the estimates of Theorem 2.5 , it is clear that $\mathcal{J}(\phi) \in C^{\infty}(\mathfrak{a})^{W}$. Next write, for $x \in \mathfrak{a}_{-}, \mathcal{J}(\phi)(x)$ in the form (3.2). Now it is obvious that we may send $p$ to infinity in $\mathfrak{a}_{+}^{*}$ and apply the usual arguments of Helgason (cf. [11], Theorem 7.3) to see that the support of $\mathcal{J}(\phi)$ indeed is contained in $C_{x}$.

\section{The inversion formula and the Plancherel formula}

We want to prove that $\mathcal{J}$ is the inverse of $\mathcal{F}$. The proof is very similar to the proof of this in the case when $k_{\alpha}>0$, as in [17], Section 9. The heart of the matter is to use Peetre's characterization of differential operators [18]. The idea to use the result of Peetre goes back to Van den Ban and Schlichtkrull [1].

Lemma 5.1. The composition $\mathcal{K}=\mathcal{J} \circ \mathcal{F}$ is formally symmetric on $C_{c}^{\infty}(\mathfrak{a})^{W}$ with respect to $(\cdot, \cdot)$ (as defined in (1.5)). Moreover, $\mathcal{K}$ commutes with the operators $D_{p}$, $p \in \operatorname{Sym}(\mathfrak{h})^{W}$.

Proof. By the previous section, $\mathcal{K}$ maps $C_{c}^{\infty}(\mathfrak{a})^{W}$ onto itself. Using Theorem 3.4, Theorem 4.1, and Fubini's theorem, we have for $f, g \in C_{c}^{\infty}(\mathfrak{a})^{W}$ :

$$
(\mathcal{K} f, g)=\int \mathcal{F}(f)(\lambda) \overline{\mathcal{F}(g)(\lambda)} d \nu(\lambda)
$$

(we used that $-c_{L} \in W\left(R_{L}\right) c_{L}$, cf. [9], Theorem 3.10.) and this is visibly a symmetric expression. The rest is trivial.

Proposition 5.2. The operator $\mathcal{K}$ is support preserving on $C_{c}^{\infty}(\mathfrak{a})^{W}$.

Proof. By Lemma 5.1 and the previous section, $\mathcal{K}$ satisfies exactly the properties which are used in the argument of [17], Lemma 9.3.

Lemma 5.3. There exists a $W$-invariant polynomial $p$ on $\mathfrak{a}^{*}$ such that $\mathcal{K}=D_{p}$.

Proof. By Peetre's theorem [18], it is clear that $\mathcal{K}$ is a differential operator on $C_{c}^{\infty}$ $\left(\mathfrak{a}^{\text {reg }}\right)^{W}$, locally of finite order and with coefficients in $C^{\infty}\left(\mathfrak{a}^{\text {reg }}\right)$. If one explicitly writes down the highest degree terms of the commutators $\left[D_{p_{i}}, \mathcal{K}\right],\left(\left\{p_{i}\right\}\right.$ a set of generators of the ring of $W$-invariant polynomials) on a suitably small open set in $\mathfrak{a}^{\text {reg }}$, one sees that the highest degree term of $\mathcal{K}$ has to have constant coefficients. Because $\mathcal{K}$ extends to a map from $C_{c}^{\infty}(\mathfrak{a})^{W}$ to itself, one concludes that the highest order term of $\mathcal{K}$ is of the form $\partial(q)$ with $q$ a $W$-invariant polynomial. Now we can replace $\mathcal{K}$ by $\mathcal{K}-D_{q}$, which still commutes with all operators of the form $D_{p}$ but has lower order than $\mathcal{K}$. By induction on the order, the result is proved.

Theorem 5.4. The maps $\mathcal{F}: C_{c}^{\infty}(\mathfrak{a})^{W} \rightarrow P W^{W}$ and $\mathcal{J}: P W^{W} \rightarrow C_{c}^{\infty}(\mathfrak{a})^{W}$ are inverse to each other.

Proof. To prove that $\mathcal{K}$ is the identity, we resort to the asymptotic formulas of Van den Ban and Schlichtkrull [1], Lemma 12.15, and Corollary 13.3 (as in [17], Lemma 9.8 and Lemma 9.9). The details are a little bit more complicated than in [17] because we have to reduce to the most continuous part of the spectrum first. Therefore, let $q$ be a nonzero $W$-invariant polynomial on $\mathfrak{h}^{*}$ that vanishes on the lower dimensional residual subspaces and $p$ as in Lemma 5.3. Then the argument of [17], Theorem 9.10 applied to $\mathcal{K} \circ D_{q}$, shows that $p q=q$; hence, $p=1$. The proof that $\mathcal{K}^{\prime}=\mathcal{F} \circ \mathcal{J}$ is the identity is completely similar to [17], Lemma 9.11 . 
Theorem 5.5 (Plancherel Theorem). The map $\mathcal{F}$ extends naturally to an isomorphism

$$
\mathcal{F}: L_{2}(\mathfrak{a}, \delta(k, x) d x)^{W} \rightarrow\left\{\bigoplus_{L \text { tempered }} L_{2}\left(L^{\text {temp }}, \nu_{L}(k)\right)\right\}^{W} .
$$

Proof. By (5.1) and Theorem 5.4, it is clear that $\mathcal{F}$ extends to an isometry defined on $L_{2}(\mathfrak{a}, \delta(k, x) d x)^{W}$. In the remaining part of the proof, we will use $\mathcal{F}$ to denote this extended map. It remains to prove the surjectivity of $\mathcal{F}$. Note that, because $\mathcal{F}$ is an isometry defined on a Hilbert space, the image of $\mathcal{F}$ is closed. This being said, we embark on the proof of the surjectivity. First we claim that if $p$ is a polynomial, nonvanishing on $i \mathfrak{a}^{*}$, then $p . P W$ restricted to $i \mathfrak{a}^{*}$ is dense in $L_{2}\left(i \mathfrak{a}^{*}, \nu_{i \mathfrak{a}^{*}}\right)$. In order to see this, recall that $P W$ is dense in $\mathcal{S}$, the ordinary Schwartz space on $i \mathfrak{a}^{*}$. If $\phi \in C_{c}^{\infty}\left(i \mathfrak{a}^{*}\right)$ and $f_{n} \rightarrow \phi$ in the Schwartz space topology, with $f_{n} \in P W$, then $p . f_{n} \rightarrow p . \phi$ in $L_{2}\left(i \mathfrak{a}^{*}, \nu_{i \mathfrak{a}^{*}}\right)$ (because the function $c(\lambda, k)^{-1}$ is bounded on $\left.i \mathfrak{a}^{*}\right)$. On the other hand, by the nonvanishing of $p, p . \phi$ is an arbitrary element in $C_{c}^{\infty}\left(i \mathfrak{a}^{*}\right)$. Whence the density statement is proven, as claimed.

There exists a $W$-invariant polynomial $p$ which is nonvanishing on $i \mathfrak{a}^{*}$, but which vanishes on all the residual subspaces other than $i \mathfrak{a}^{*}$ (see [9], proof of Lemma 3.1, uniqueness part). It is clear, using Theorem 5.4, that the image of $\mathcal{F}$ contains p.PWW (where $p$ is the invariant polynomial just mentioned), and the closure of this in the right-hand side of (5.2) equals $L_{2}\left(i \mathfrak{a}^{*}, \nu_{i \mathfrak{a}^{*}}\right)^{W}$, by the above argument. Hence, the summand $L_{2}\left(i \mathfrak{a}^{*}, \nu_{i \mathfrak{a}^{*}}\right)^{W}$ of the right-hand side of (5.2) is in the image of $\mathcal{F}$. Now we argue by induction on the length $\left|c_{L}\right|$ of a center $c_{L}$ of a residual subspace $L$. Assume that all the summands of the right-hand side of (5.2) that correspond to residual subspaces with center $c$ such that $|c|<\left|c_{L}\right|$ are in the image of $\mathcal{F}$. Let $p$ be a $W$ invariant polynomial which vanishes on all residual subspaces with center $c$ such that $|c| \geq\left|c_{L}\right|$ but $c \notin W c_{L}$, and nonvanishing on $W\left(c_{L}+i \mathfrak{a}^{*}\right)$ (again, such a polynomial exists by [9], proof of Lemma 3.1, uniqueness part). It follows that the restriction of $p . P W^{W}$ to the union of the tempered forms $L_{1}^{\text {temp }}, \ldots, L_{l}^{\text {temp }}$ of the residual subspaces contained in $W\left(c_{L}+i \mathfrak{a}^{*}\right)$ is in the image of $\mathcal{F}$. Call this space of functions $\Sigma_{L}$. Let $\phi$ be a $W$-invariant $C_{c}^{\infty}$ function on the $W$ orbit $W\left(c_{L}+i \mathfrak{a}^{*}\right)$, and $\phi^{\prime}$ its restriction to the union of $L_{1}^{\text {temp }}, \ldots, L_{l}^{\text {temp }}$. As before, $\phi$ can be approximated, with respect to the topology of the direct sum of the Schwartz spaces of functions on the $W$-translates of $c_{L}+i \mathfrak{a}^{*}$, by elements from $p . P W^{W}$ (restricted to $W\left(c_{L}+i \mathfrak{a}^{*}\right)$ ). This implies the approximation of $\phi^{\prime}$ by elements of $\Sigma_{L}$ because (3.7) and Theorem 3.4 show that the Plancherel measure $\nu_{L_{i}(k)}$ is given by a polynomially bounded measure which is smooth on $L_{i}^{\text {temp }}$. Finally, it is clear that the functions $\phi^{\prime}$ form a dense subspace of $\left\{\oplus L_{2}\left(L_{i}^{\text {temp }}, \nu_{L_{i}(k)}\right)\right\}^{W}$ because of the smoothness of $\nu_{L_{i}(k)}$ on $L_{i}^{\text {temp }}$. This completes the induction step.

Remark 5.6. This argument also works in the context of the Lieb-McGuire system of particles studied in [9], cf. Remark 3.21 of that paper.

Theorem 5.7. The $W$-invariant, square integrable (or cuspidal) eigenfunctions of $L(k)$ are precisely those of the form $F(\lambda, k)$ with $\lambda$ a cuspidal point. (The cuspidal points are classified in [9], Section 4. There are a finite number of such points for each root system and each multiplicity function $k$ satisfying the conditions of Proposition 1.1.) 
Proof. First of all, by the classification of the dominant cuspidal points in [9], it is easy to check that these are ordered by the dominance ordering. In particular, the $L(k)$ eigenvalues are separated. We already know that the corresponding hypergeometric functions are square integrable by Corollary 3.7. It remains to show that these are the only $L_{2}$ eigenfunctions, but this is obvious from Theorem 5.5 since the measures $\nu_{L}$ are absolutely continuous with respect to the Lebesgue measure on $L^{\text {temp }}$.

Corollary 5.8. Let $\lambda=L$ be a cuspidal point, for some multiplicity function $k_{0} \in \mathcal{K}$. There exists at least one linear parameter family of $\left(\mathfrak{a}^{*}, R, k\right)$-distinguished points $\lambda(k)$ such that $\lambda\left(k_{0}\right)=\lambda$ and such that $\lambda(k)$ is cuspidal when $k$ is in an open simplex $\Sigma \subset \mathcal{K}$ containing $k_{0}$. In the simply laced case, this simplex is always equal to $\left(-\frac{1}{d_{n}}, 0\right)$ (with $d_{n}$ the Coxeter number of $W$ ) and, in the other cases, $\Sigma$ is bounded by two lines through $0 \in \mathcal{K}$ and the line $\rho(k)\left(\beta^{\vee}\right)+k_{\beta}+1=0$ (as in (1.6)). The function $F(\lambda(k), k ; \cdot)$ is square-integrable for all $k$ in $\Sigma+i \mathcal{K}$. Let $R_{z}=\left\{\alpha \in R \mid \lambda(k)\left(\alpha^{\vee}\right)=0 \forall k \in \mathcal{K}\right\}$ and let $R_{p}=\left\{\alpha \in R \mid \lambda(k)\left(\alpha^{\vee}\right)+k_{\alpha}=0 \forall k \in \mathcal{K}\right\}$. Then

$$
\int_{\mathfrak{a}} F(\lambda(k), k)^{2} \delta(k, x) d x=c \frac{\prod_{\alpha \in R_{+}} \Gamma\left(\rho(k)\left(\alpha^{\vee}\right)+k_{\alpha}\right)^{2} \prod_{\alpha \in R \backslash R_{z}} \Gamma\left(\lambda(k)\left(\alpha^{\vee}\right)\right)}{\prod_{\alpha \in R_{+}} \Gamma\left(\rho(k)\left(\alpha^{\vee}\right)\right)^{2} \prod_{\alpha \in R \backslash R_{p}} \Gamma\left(\lambda(k)\left(\alpha^{\vee}\right)+k_{\alpha}\right)} .
$$

Here the constant $c$ is rational and of the form

$$
c= \pm \gamma_{L}^{-1}(k)|W \lambda(k)|^{-1}
$$

(cf. Theorem 3.4 for the definition of the rational number $\gamma_{L}(k)$ ) where $k \in \Sigma$ is a generic point.

Proof. The process of taking residues of

$$
\int_{p+i \mathfrak{a}^{*}} \phi(\lambda) \frac{d \lambda}{c_{Y}(\lambda, k), c_{Y}(-\lambda, k)}
$$

as described in [9], is continuous with respect to $k$ in the sense that the local contribution at $\lambda$ equals the sum of the limits of the local contributions at the cuspidal points $\lambda^{\prime}(k)$ which have the property that $\lambda^{\prime}(k) \rightarrow \lambda$ as $k \rightarrow k_{0}$ along a generic line. To see this, first note that (5.4) is continuous in $k$. Then one checks that the individual limits of the local contributions at the $\lambda(k)$ exist, by inequality (3.17) of [9]. Thus, one now may use the uniqueness property of local contributions (cf. [9], Lemma (3.1)) to conclude the asserted continuity. Hence, there exists at least one linear family $\lambda(k)$ of cuspidal points as stated in the theorem. The polynomials $p_{\mu, \kappa}(x ; k)$ in the asymptotic expansion (3.12) of $F(\lambda(k), k, \cdot)$ are meromorphic in $k$, and their joint sets of singularities form an analytic subset of $\mathcal{K}_{c}$. Therefore, the set of leading terms of $F(\lambda(k), k, \cdot)$ at any specialization of $k \in \mathcal{K}_{c}$ is contained in the specialization of the generic set of exponents, and this generic set is of the form $\{w \lambda(k) \mid w \in D\}$ for some $D \subset W$. Moreover, on an arbitrary, sufficiently small compact torus $T$ in $\mathcal{K}_{c}$, with center $k_{1}$ say, which avoids the singularities of the $p_{\mu, \kappa}(x ; k)$, the estimate (3.13) holds uniformly: $\forall k \in T \forall x \in \overline{\mathfrak{a}_{-}}$:

$$
|F(\lambda(k), k ; x)| \leq M(1+|x|)^{m} e^{\tau(x)}
$$

for some $\tau>\rho\left(k_{1}\right)$. Hence, by Cauchy's theorem, the same type of estimate holds for derivatives of $F(\lambda(k), k ; x)$ with respect to $k$, and we conclude that the left-hand side of (5.3) is a holomorphic function in the tube $\left\{k \in \mathcal{K}_{c} \mid \operatorname{Re}(w \lambda(k))>0 \forall w \in D\right\}$ $\cap\left\{k \in \mathcal{K}_{c} \mid \operatorname{Re}(k)\right.$ satisfies (1.6) $\}$. This set is of the form $\Sigma+i \mathcal{K}$ with $\Sigma$ a simplex 
as described in the theorem. We now compute the integral explicitly when $k \in \Sigma$ and generic by applying $\mathcal{F}$ to $F(\lambda(k), k)$ and using Theorem 5.5 . The rest of the statement follows by analytic continuation.

The following special case was obtained previously in [2], using different methods.

Example 5.9. Take $\lambda(k)=\rho(k)$ in the previous theorem. In this situation, we know that $F(\rho(k), k ; x)=1$, and thus $\Sigma=\{k \in \mathcal{K} \mid k$ satisfies (1.6) $\}$. The constant $c$ equals $|W|$, and if one takes $k_{\alpha}=k \forall \alpha \in R$, one obtains

$$
\int_{\mathfrak{a}} \delta(k, x) d x=\prod_{i=1}^{n}\left(\begin{array}{c}
d_{i} k \\
k
\end{array}\right) \frac{\pi}{\sin \left(-m_{i} \pi k\right)}
$$

where the $d_{i}$ denote the primitive degrees of $W$, and the $m_{i}$ are the exponents of $W$.

Acknowledgment. I thank Patrick Delorme and Gerrit Heckman for many helpful conversations and comments.

\section{References}

1. E. P. van den Ban and H. Schlichtkrull, The most continuous part of the Plancherel decomposition for a reductive symmetric space, Preprint series, University of Utrecht 921, 1995.

2. R. Brusse, G. J. Heckman, and E. M. Opdam, Variation on a theme of Macdonald, Math. Z. 208 (1991), 1-10.

3. W. Casselman and D. Miličić, Asymptotic behavior of matrix coefficients of admissible representations, Duke Math. Jour. 49 (1982), 869-930.

4. I. Cherednik, A unification of Knizhnik-Zamolodchikov equations and Dunkl operators via affine Hecke algebras, Inv. Math. 106 (1991), 411-432.

5. C. F. Dunkl, Differential-difference operators associated to reflection groups, Trans. AMS 311 (1989).

6. Harish-Chandra, Spherical functions on a semisimple Lie group I, Amer. J. Math. 80 (1958), 241-310.

7. G. J. Heckman, An elementary approach to the hypergeometric shift operators of Opdam, Inv. Math. 103 (1991), 341-350.

8. G. J. Heckman and E. M. Opdam, Root systems and hypergeometric functions I, Comp. Math. 64 (1987), 329-352.

9. __ Yang's system of particles and Hecke algebras, Annals of Mathematics 145 (1997), 139-173.

10. G. J. Heckman and H. Schlichtkrull, Harmonic Analysis and Special Functions on Symmetric Spaces, Perspectives in Mathematics 16, Academic Press, 1995.

11. S. Helgason, Groups and Geometric Analysis, Academic Press, 1984.

12. T. H. Koornwinder, Orthogonal polynomials in two variables which are eigenfunctions of two algebraically independent partial differential operators I-IV, Indag. Math. 36 (1974), 48-66; 358-381.

13. I. G. Macdonald, A formal identity for affine root systems, preprint (1996).

14. J. Moser, Three integrable Hamiltonian systems connected with isospectral deformation, Adv. Math. 16 (1975), 197-220.

15. M. A. Olshanetsky and A. M. Perelemov, Completely integrable Hamiltonian systems connected with semi-simple Lie algebras, Inv. Math. 37 (1976), 93-108.

16. E. M. Opdam, Some applications of hypergeometric shift operators, Inv. Math. 98 (1989), 1-18. 
17. - Harmonic analysis for certain representations of graded Hecke algebras, Acta Mathematica 175 (1995), 75-121.

18. J. Peetre, Rectification à l'article "Une caractérisationabstraite des opérateurs différentiels", Math. Scand. 8 (1960), 116-120.

Department of Mathematics, University of Leiden, P.O. Box 9512, 2300 RA Leiden, The NETHERLANDS 